\title{
Generalized second price auction is optimal for discrete types
}

\author{
Halil I. Bayrak, Mustafa Ç. Pınar* \\ Department of Industrial Engineering, Bilkent University, 06800 Ankara, Turkey
}

\section{H I G H L I G H T S}

- The Generalized Second Price Auction is commonly used by search engines.

- The Generalized Second Price Auction is shown to be optimal for the sale of a good.

- The result is based on linear programming duality and submodular minimization.

\section{A R T I C L E I N F O}

\section{Article history:}

Received 31 August 2015

Received in revised form

30 December 2015

Accepted 15 January 2016

Available online 9 February 2016

\section{JEL classification:}

C61

D44

Keywords:

Optimal auction design

Second price auction with reserve

Linear programming

Submodular functions

Implementation

Online advertising

\begin{abstract}
A B S T R A C T
We prove that a variant of the second price auction for the sale of a single good through a Bayesian incentive compatible mechanism that maximizes expected revenue of the seller is optimal when the type space is discrete. Moreover, we show that this variant is related to the widely used generalized second price auction mechanism in keyword-auctions for advertising, thus providing a theoretical justification for a practical tool.
\end{abstract}

(C) 2016 Elsevier B.V. All rights reserved.

\section{Introduction}

A common tool used by search engines for online keyword advertising is the generalized second price (GSP) auction, the properties of which have been analyzed by Edelman et al. (2007). In the simplest version of GSP, a bidder in the $i$ th position would pay the bid of the bidder in the $(i+1)$-th position plus a surcharge. The GSP mechanism generates billions of dollars of revenues for internet companies as documented in Edelman et al. (2007).

In a seemingly unrelated line of work, and as a culmination of fifteen years of research Vohra $(2012,2011)$ established the discrete types analogs of the celebrated optimal auction results by Myerson (1981) for an expected revenue maximizing seller using well-known tools from linear programming duality and submodular optimization.

\footnotetext{
* Corresponding author.

E-mail address: mustafap@bilkent.edu.tr (M.Ç. Pınar).
}

The purpose of this note is to show that a variant of the second price auction is optimal when the type space is discrete by making precise a statement by Vohra in Vohra (2012) which advocates a possible implementation of an optimal auction in a Myerson framework with discrete valuations of buyers for the sale of a single good, and to connect this thread to the popular GSP mechanism. More precisely, the claim was as follows (see p. 296 of Vohra, 2012):

....This is not the only implementation. There are other implementations that achieve the same interim allocation probabilities and expected payments. One of these is a second price auction with reserve. That is, the highest bidder wins, but pays the larger of the second highest bid and the reserve. In the event the highest bid does not exceed the reserve, the seller withholds the good.

We show that one should exercise some care in using this statement as it requires an alteration. We shall do so by first summarizing briefly the result of Vohra (2012), and then examining the above statement. Using the modern tools of Vohra (2012) that 
are becoming the new standard in the profession we establish a suitable adjustment of the claim, namely a scheme called the "discrete second price auction", a version of which is precisely the GSP mechanism. Thus, our work provides an easy to follow theoretical justification to the optimality of the generalized second price auction. We also develop a quantization of valuations that makes a (true) second price auction implementation possible in a discrete type space framework in the limit.

Our result is also closely related to Harris and Raviv (1981) which considered the design of an optimal auction for discrete type spaces (an equally spaced set of types) with two bidders and a revenue maximizing seller. It turns out that the optimal mechanism they obtain is also a discrete second price auction (or a generalized second price auction). Using the tools of the present paper, we also arrive at a multiple bidder version of their result obtained using a different method for two bidders. The fact that discrete types affect the insights obtained in continuous type spaces was also the central point of Lovejoy (2006) more recently, where similar observations were made in a more involved setting of optimal mechanism design with quasi-linear utilities, utilizing the concept of supermodularity.

\section{The setting}

We use exactly the setting of Vohra (2012, 2011), and sketch only the main ideas. For the details, the reader is referred to these two sources.

A risk neutral seller with a single good is facing $n$ risk neutral buyers that have non-negative private valuation for the good. These private valuations will be addressed as "agents' types" and valuation of the seller is assumed to be equal to 0 . We define $T=\{1,2, \ldots, m\}$ as the type space whose discrete form will allow the use of linear programming. The probability that an agent is of type $t$ is commonly known, and is denoted by $f_{t}>0$ for all $t \in T$. The Revelation Principle allows to focus on direct mechanisms only (see e.g., Myerson, 1981).

We use $t \in T^{n}$ to denote a profile vector. The symbols $a$ and $p$ are defined to be allocation and payment rule, respectively. The symmetry assumption allows focusing on one agent, say agent 1 . We use $a_{i}\left(i, t^{-1}\right)$ for the allocation to agent 1 , and $p_{i}\left(i, t^{-1}\right)$ is the payment done by agent 1 when she reports her type as $i \in T$ and all other agents report $t^{-1}$. We use $\pi\left(t^{-1}\right)$ for the probability of agents having types that give rise to the profile $t^{-1}$. The number of agents with type $i$ in profile $t$ is shown by $n_{i}(t)$. Interim (expected) allocation and payment are denoted accordingly when all agents other than agent 1 report their type truthfully:

$$
\begin{aligned}
& A(i)=\sum_{t^{-1} \in T^{n-1}} a_{i}\left(i, t^{-1}\right) \pi\left(t^{-1}\right), \\
& P(i)=\sum_{t^{-1} \in T^{n-1}} p_{i}\left(i, t^{-1}\right) \pi\left(t^{-1}\right) .
\end{aligned}
$$

The objective of the problem is to maximize the seller's expected revenue, and we face the following optimization problem:

$$
\begin{aligned}
& \max _{P, A, a} \sum_{i \in T} f_{i} P(i) \\
& \text { s.t. } \quad i A(i)-P(i) \geq i A(j)-P(j) \\
& i A(i)-P(i) \geq 0 \\
& \forall i, j \in T \\
& A(i)=\sum_{t^{-1} \in T^{n-1}} a_{i}\left(i, t^{-1}\right) \pi\left(t^{-1}\right) \\
& \sum_{i \in T} n_{i}(t) a_{i}(t) \leq 1 \\
& \forall t \in T^{n} \\
& a_{i}(t) \geq 0 \\
& \forall i \in T, \forall t \in T^{n} \text {. }
\end{aligned}
$$

Obviously, constraints (4) and (5) ensure that only one good is allocated for each profile and no agent receives a negative amount while constraint (3) only relates interim allocation variables to allocation rule variables. Constraint (2) expresses individual rationality, and constraint (1) is the Bayes-Nash Incentive Compatibility (BNIC) constraint. It is clear that we are only interested in the mechanisms in which the optimal strategy is to report truthfully.

\section{The solution}

Consider the system of constraints (1) and (2). The following is well-known:

Theorem 1. System (1) is feasible if and only if interim allocations are monotonic. That is, if $i \leq j$, then $A(i) \leq A(j)$.

Then, to maximize expected revenue one sets each $P(i)$ to its upper bound which is equal to $\sum_{k=1}^{i} k A(k)-k A(k-1)$ (the upper bound is calculated using the result of the above theorem). Define $v(i)=i-\frac{1-F(i)}{f_{i}}$. If the hazard function is monotone then $v(i)$ is non-decreasing in $i$. Now the formulation is:

$$
\begin{aligned}
& \max _{A, a} \sum_{i \in T} f_{i} v(i) A(i) \\
& \text { s.t. } \quad 0 \leq A(1) \leq \cdots \leq A(m) \\
& A(i)=\sum_{t^{-1} \in T^{n-1}} a_{i}\left(i, t^{-1}\right) \pi\left(t^{-1}\right) \quad \forall i \in T \\
& \sum_{i \in T} n_{i}(t) a_{i}(t) \leq 1 \quad \forall t \in T^{n} \\
& a_{i}(t) \geq 0 \quad \forall i \in T, \forall t \in T^{n} .
\end{aligned}
$$

At this point, one takes out $a_{i}(t)$ variables in order to end up with a polymatroid optimization problem as a result of the following theorem.

Theorem 2 (Border's Theorem). The expected allocation $A(i)$ is feasible if and only if

$$
n \sum_{i \in S} f_{i} A(i) \leq 1-\left(\sum_{i \notin S} f_{i}\right)^{n} \quad \forall S \subseteq T .
$$

Using this theorem the reduced formulation becomes:

$$
\begin{array}{ll}
\max _{A} & \sum_{i \in T} f_{i} v(i) A(i) \\
\text { s.t. } & 0 \leq A(1) \leq \cdots \leq A(m) \\
& n \sum_{i \in S} f_{i} A(i) \leq 1-\left(\sum_{i \notin S} f_{i}\right)^{n} \quad \forall S \subseteq T .
\end{array}
$$

Then, defining the function $G(S)$ which is nondecreasing, nonnegative and submodular, and setting $x_{i}=f_{i} A(i)$ for all $i \in T$ we get the problem

$$
\begin{array}{lll}
\max _{A} & \sum_{i \in T} v(i) x_{i} & (\text { OPTb }) \\
\text { s.t. } & 0 \leq \frac{x_{1}}{f_{1}} \leq \cdots \leq \frac{x_{m}}{f_{m}} & \\
& \sum_{i \in S} x_{i} \leq G(S)=\frac{1-\left(\sum_{i \notin S} f_{i}\right)^{n}}{n} \quad \forall S \subseteq T .
\end{array}
$$

Ignoring the monotonicity constraint, this becomes a polymatroid optimization problem which is solved optimally by the Greedy Algorithm. Under the monotone $v(i)$ function assumption, the 
optimal solution is as follows: when $i^{*}$ is the lowest type such that $v\left(i^{*}\right) \geq 0$ :

$A(i)= \begin{cases}\frac{F(i)^{n}-F(i-1)^{n}}{f_{i} n} & \text { if } i \geq i^{*} \\ 0 & \text { otherwise. }\end{cases}$

It is easy to check that this solution for $A(i)$ satisfies the monotonicity constraint. Therefore it is optimal for $O P T b$.

\section{Optimal payment rule}

As quoted in the introduction, the second price auction is said to achieve the same interim allocation and expected payment values as the optimal solution of OPTb. However, we shall see below that this is not the case. Recall the optimal solution for the expected payments:

$P(i)=\sum_{k=1}^{i} k A(k)-k A(k-1)=i A(i)-\sum_{k=1}^{i} A(k-1)$.

Denote the underlying payment rule by $p_{i}\left(i, t^{-1}\right)$ which is the payment that agent 1 makes when he reports $i$ and others report $t^{-1}$. It is easily verified that the following setting is consistent with the expected payments, i.e., defining the payment rule

$p_{i}\left(i, t^{-1}\right)=i a_{i}\left(i, t^{-1}\right)-\sum_{k=1}^{i} a_{k-1}\left(k-1, t^{-1}\right)$,

and taking its expectation we find exactly the $P(i)$ as given above.

Consider now the allocation rule in which we allocate the good to the highest bidder if he is above the reserve price $i^{*}$. Vohra asserts correctly that this allocation rule is consistent with the optimal interim allocations. However, when we use this allocation rule to find the optimal price rule from (6), we find a payment scheme different from the second price auction.

Example 1. We have two agents and our type set is $T=$ $\{1,2, \ldots, 10\}$. Consider the allocation rule where the reserve price is 6 . Then we can calculate the payment of winner in profile $(8,2)$ as 6 since the winner would not change if he bid 7 or 6 . However, for the profile $(8,6)$ payment is different from the second price auction:

$$
\begin{aligned}
p_{8}(8,6) & =8 \cdot a_{8}(8,6)-a_{7}(7,6)-a_{6}(6,6) \\
& =8-1-0.5=6.5 .
\end{aligned}
$$

We call our mechanism the "discrete second price auction" (DSP) where the second highest bid is defined as $s=\max _{k \in t^{-1}} k$. The resulting formulae are:

$$
\begin{aligned}
& a_{i}^{*}\left(i, t^{-1}\right)= \begin{cases}\frac{1}{n_{i}(t)} & \text { if } i \geq \max _{k \in t^{-1}} k \geq i^{*} \\
0 & \text { otherwise }\end{cases} \\
& p_{i}^{*}\left(i, t^{-1}\right)= \begin{cases}\frac{i}{n_{i}(t)} & \text { if } i=\max _{k \in t^{-1}} k \geq i^{*} \\
s+\frac{n_{s}\left(t^{-1}\right)}{n_{s}\left(t^{-1}\right)+1} & \text { if } i>s=\max _{k \in t^{-1}} k \geq i^{*} \\
i^{*} & \text { if } i \geq i^{*}>\max _{k \in t^{-1}} k \\
0 & \text { otherwise. }\end{cases}
\end{aligned}
$$

The DSP auction scheme is akin to the Generalized Second Price (GSP) auction scheme used in keyword auctions for web advertising (Edelman et al., 2007). In the simplest GSP auction, an advertiser in position $i$ pays a price per click equal to the bid of an advertiser in position $(i+1)$ plus a minimum increment (typically \$0.01).

Actually, the difference between the second price and the discrete second price auctions is a result of the assumption that the type set is discrete. The second price auction defined in Myerson does not have to deal with the case where some agents share the good because this case has zero probability when the type set is continuous. However, we have this case with positive probability, and the seller can improve his expected revenue by exploiting the sole winner. After all, the sole winner does not share the good with the second highest bidders. Note also that as the number of the agents who are willing to pay the second highest bid increases, payment of the sole winner increases, and converges to the type just above the second highest bid.

A similar mechanism is contained in Theorem 2 of Harris and Raviv (1981) for the case of two bidders, although the notation is slightly different, and a different methodology is used. However, taking the increment between two consecutive valuations in Theorem 2 of Harris and Raviv (1981) equal to one, we find exactly the result above. We generalize this result below to fit the valuations representation in Harris and Raviv (1981). We note that Lovejoy (2006) also obtained the same result as a special case of a more general result (see Corollary 5 and the ensuing discussion in Lovejoy, 2006).

Now consider a slightly different setting where we allow buyers to make bids only in the multiples of $q$ where $0<q<1$ as in Harris and Raviv (1981). Again we will work on a finite set so we set $M$ as the highest bid such that $M=m \cdot q$. Our type set is the same as before, namely $T=\{1,2, \ldots, m\}$, however this time, type $i$ actually refers to a bid equal to $i \cdot q$, i.e., the type space can be considered as $\{q, 2 q, \ldots, m q\}$. We will have the following changes in the formulation:

$$
\begin{aligned}
& \max _{P, A, a} \sum_{i \in T} f_{i} P(i) \\
& \text { s.t. } \quad q i A(i)-q P(i) \geq q i A(j)-q P(j) \\
& \forall i, j \in T \\
& q i A(i)-q P(i) \geq 0 \\
& \forall i \in T \\
& A(i)=\sum_{t^{-1} \in T^{n-1}} a_{i}\left(i, t^{-1}\right) \pi\left(t^{-1}\right) \\
& \forall i \in T \\
& \sum_{i \in T} n_{i}(t) a_{i}(t) \leq 1 \\
& \forall t \in T^{n} \\
& a_{i}(t) \geq 0 \\
& \forall i \in T, \forall t \in T^{n} .
\end{aligned}
$$

In the constraints (1) and (2), $q$ cancels out, and since it is a scalar we can also remove it from the objective. Under the same assumptions on the probability mass $f$, the optimal solution will be almost identical to the one given above, with a slight difference in the payment rule:

$$
p_{i}\left(i, t^{-1}\right)= \begin{cases}q \cdot \frac{i}{n_{i}(t)} & \text { if } i=\max _{k \in t^{-1}} k \geq i^{*} \\ q \cdot s+q \cdot \frac{n_{s}\left(t^{-1}\right)}{n_{s}\left(t^{-1}\right)+1} & \text { if } i>s=\max _{k \in t^{-1}} k \geq i^{*} \\ q \cdot i^{*} & \text { if } i \geq i^{*}>\max _{k \in t^{-1}} k \\ 0 & \text { otherwise. }\end{cases}
$$

Next we show that taking the limit of this payment rule as $q$ approaches to 0 will give us the second price auction. Before that we need to rewrite the payment rule in a different way since as $q$ changes our type set will also change. Take a $j$ value which is a multiple of $q$ and less than $M$ so that buyers can have it as their bid. Then there exists a type $j^{\prime} \in T$ such that $j=j^{\prime} \cdot q$ : (define $s$ and 
$i^{*}$ in the same way). The payment rule is now

$p_{j^{\prime}}\left(j^{\prime}, t^{-1}\right)$

$$
= \begin{cases}q \cdot \frac{j}{q} \cdot \frac{1}{n_{j^{\prime}}(t)} & \text { if } j^{\prime}=\max _{k \in t^{-1}} k \geq i^{*} \\ q \cdot \frac{s}{q}+q \cdot \frac{n_{s^{\prime}}\left(t^{-1}\right)}{n_{s^{\prime}}\left(t^{-1}\right)+1} & \text { if } j^{\prime}>s^{\prime}=\max _{k \in t^{-1}} k \geq i^{*} \\ q \cdot \frac{i^{*}}{q} & \text { if } j^{\prime} \geq i^{*^{\prime}}>\max _{k \in t^{-1}} k \\ 0 & \text { otherwise. }\end{cases}
$$

As $q$ tends to 0 , the type set should also change in order to satisfy $j=j^{\prime} \cdot q$ for any positive $j \leq M$. Clearly, the only point that made our optimal payment rule different from the second price auction now vanished. Then one can assert that the optimal solution obtained using linear programming is fully consistent with the results of Myerson. The result given above is thus a simple multiple agent version of Theorem 2 of Harris and Raviv (1981) for two bidders.

\section{Acknowledgment}

Special thanks to Kemal Güler for providing valuable input.

\section{References}

Edelman, B., Ostrovsky, M., Schwarz, M., 2007. Internet advertising and the generalized second price auction: Selling billions of dollars worth of keywords. Amer. Econ. Rev. 97 (1), 242-259.

Harris, M., Raviv, A., 1981. Allocation mechanisms and the design of auctions. Econometrica 49 (6), 1477-1499.

Lovejoy, W., 2006. Optimal mechanisms with finite agent types. Manage. Sci. 52 (5), $788-803$.

Myerson, R.B., 1981. Optimal auction design. Math. Oper. Res. 6 (1), 58-73.

Vohra, R.V., 2011. Mechanism Design: A Linear Programming Approach, Vol. 47. Cambridge University Press.

Vohra, R.V., 2012. Optimization and mechanism design. Math. Program. 134 (1), 283-303. 